\title{
Title: Infective Endocarditis in Low- And Middle-Income Countries
}

\section{Authors:}

Name:

Degree:

Affiliation:

Email:

Mailing address:

Disclosure statement:

Name:

Degree:

Affiliation:

Email:

Mailing address:

Disclosure statement:

Name:

Degree:

Affiliation:

Email:

Mailing address:

Disclosure statement:

Name:

Degree:

Affiliation:

Email:

Mailing address:

Disclosure statement:
Benson Njuguna, BPharm

Bachelor of Pharmacy

Moi Teaching and Referral Hospital, Eldoret, Kenya

njugunaben1@gmail.com

PO Box 5760 Eldoret 30100, Kenya

The author has nothing to disclose

Adrian Gardner, MD, MPH

Doctor of Medicine, Master of Public Health

Indiana University School of Medicine, Indiana, USA

gardner5@iu.edu

PO Box 5760 Eldoret 30100, Kenya

The author has nothing to disclose

\section{Rakhi Karwa, PharmD, BCPS}

Doctor of Pharmacy, Board Certified Pharmacotherapy Specialist

Purdue University College of Pharmacy

rkarwa@purdue.edu

PO Box 5760 Eldoret 30100, Kenya

The author has nothing to disclose

\section{François DELAHAYE, MD, PhD}

Doctor of Medicine, Doctor of Philosophy

Hospices civils de Lyon; Université Claude Bernard, Equipe d'Accueil HESPER 7425

francois.delahaye@univ-lyon1.fr

Hôpital Louis Pradel, 28, avenue du Doyen Lépine, 69677 - Bron Cedex, France

Corresponding Author:

Benson Njuguna

This is the author's manuscript of the article published in final edited form as: 
Key Words: endocarditis in LMIC, developing countries, causes, management, challenges

\section{Key Points}

- Staphylococcus is an increasingly important cause of IE in LMICs, and is the leading cause of IE in UMICs

- RHD remains the major underlying cardiac pathology of IE in LMICs, identified in almost half of reported cases

- The rate of microorganism non-identification is high, reaching up to $60 \%$ of IE cases in LMICs, and hampering diagnosis and treatment

- Rates of access to surgery in UMICs for complicated IE are as high as in HICs, but remain dismal in lower-middle income countries

\section{Synopsis}

Infective endocarditis (IE) is a rare, life-threatening disease with a mortality rate of upto $25 \%$ and significant debilitating morbidities. Although much has been reported on contemporary IE in high income countries, conclusions on the state of IE in low and middle income countries (LMICs) are based on studies conducted before the year 2000. Furthermore, unique challenges in the diagnosis and management of IE persist in LMICs. This article is a review of IE studies conducted in LMICs documenting clinical experiences from the year 2000 to present. We present the causes of IE, management of patients with IE and the prevailing challenges in diagnosis and treatment of IE in LMICs. 


\section{Infective endocarditis in low-and middle-income countries}

\section{Introduction}

Infective endocarditis (IE) is a rare, life-threatening disease with a significant mortality and morbidity burden. In-hospital mortality approaches $25 \%$, increasing in patients with cardiac or extracardiac complications. ${ }^{1-3}$ IE also frequently causes debilitating morbidities, such as heart failure, stroke and renal failure requiring dialysis, which contribute to increased mortality and disability adjusted life years (DALYs). ${ }^{3-7}$

The spectrum of causative microorganisms and underlying risk factors for IE has shifted dramatically in high income countries (HICs). ${ }^{8,9}$ Staphylococcal IE now predominates. Degenerative valve disease (DVD), prosthetic valves and other intracardiac devices are the leading underlying cardiac conditions, with little contribution from rheumatic heart disease (RHD) and congenital heart disease (CHD).

IE in low and middle-income countries (LMICs) has been said to resemble that seen in HIC-based studies from the mid- $20^{\text {th }}$ century which reported a predominance of IE due to streptococcal infection, RHD and CHD as the leading risk factors, and minimal rates of surgical intervention. ${ }^{9}$ Reports from LMICs that mirror this epidemiology and treatment patterns are predominantly from before the turn of this millennium and consequently, may not reflect the current state of IE in LMICs. ${ }^{10-14}$

The prevalence of RHD remains disproportionately high in LMICs, while uncorrected CHD persists in the poorest of these settings due to limited access to cardiac surgery. ${ }^{15,16}$ It is therefore expected that RHD and CHD remain significant underlying cardiac conditions for IE; however, economic improvement in lower-middle income and upper-middle income 
countries has led to medical progress which may have introduced additional IE risk factors for these populations, and altered the spectrum of causative microorganisms.

In this review, we present the causes and treatment of IE in LMICs as reported in contemporary studies, defined as studies which primarily report findings from the year 2000 and after. We also discuss the prevailing challenges to the diagnosis and management of IE in LMICs, and suggest future directions in research.

\section{Methods}

We searched PUBMED and EMBASE using the keywords: endocarditis, infective endocarditis, low income, middle income, and developing country. LMICs and HICs were based on 2016 World Bank Income groups classification. ${ }^{17}$ Articles were considered relevant if they were original research that described IE epidemiology and management experiences from LMICs from the year 2000 and after. We also screened the reference list of the retrieved articles for additional relevant studies.

\section{CAUSES OF IE}

Degenerative valve disease (DVD), prosthetic valves, and intracardiac electronic devices have replaced RHD as the major underlying cardiac risk factors for IE in HICs. ${ }^{1}$ Comorbidities such as diabetes mellitus (DM), renal failure requiring dialysis, and malignancy contribute substantially to a growing burden of healthcare-associated IE

(HAIE). ${ }^{2,18}$ Consequently, growing use of long-term intra-vascular access devices has led to skin bacteria in the form of Staphylococcus being the leading cause of IE in HICs. ${ }^{1,2,18}$

In this section, we review the prevalent underlying cardiac conditions, place of acquisition and microbial etiology of IE in LMICs. A summary of our findings is presented in Table 1. 


\section{Underlying cardiac conditions}

Rheumatic heart disease (RHD) is identified as the underlying cardiac pathology in a majority of IE cases, ranging from $28-45 \%$ in most of our reviewed studies. ${ }^{19-24} \mathrm{Nel}$ et al. ${ }^{25}$ however, reported a much higher (78\%) prevalence of underlying RHD in their study in South Africa, a country where RHD is endemic. ${ }^{26}$

Overall, this range of underlying RHD in IE represents a decline from the $45-80 \%$ reported in earlier (pre-2000) LMIC studies. ${ }^{10,11,13,27,28}$ Compared to HICs however, these findings are remarkable in that RHD is identified in only $3 \%$ of IE cases. ${ }^{1}$ This is likely because the prevalence of RHD is disproportionately high in $\mathrm{LMICs},{ }^{29,30}$ which bear $79 \%$ of the global RHD burden. ${ }^{15}$

Underlying CHD accounts for 5-23\% of reported IE cases. ${ }^{19,21,23-25,31,32}$ Math et al. ${ }^{20}$ reported CHD as the leading cause of IE (39\%) in northern India. This study was conducted in a cardiac surgery centre and included a large paediatric population which may account for the higher findings of CHD.

Prosthetic valve IE (PVE) and pacemakers/intracardiac defibrillators is reported in 17$44 \%$ and $6-19 \%$ of IE cases in LMICs respectively. ${ }^{19-23,31,32}$ UMIC studies account for the higher figures in these ranges, which reflects advances in medical technology and higher access to cardiac surgery. ${ }^{21,22}$ Indeed, the largest report of PVE cases from a LMIC comes from Simsek-Yavuz et al. ${ }^{21}$ who reported findings from a referral centre in Turkey (an UMIC) in which 141 patients (44\%) among 325 IE cases had PVE. 52\% of the total patient cohort received surgical intervention, closely approximating the rate seen in HICs. ${ }^{2}$

Degenerative valve disease (DVD) contributes little to the IE epidemiology in LMICs, accounting for less than $10 \%$ of cases. ${ }^{19,21,23,32}$ Elbey et al. ${ }^{31}$ however, reported a high rate of 
DVD (23\%) in a multicentre retrospective study in Turkey. Mean patient age was 47 years, higher than that typically seen in LMIC studies, which may explain the higher rate of DVD. Overall, unlike in HICs where DVD is the major underlying cardiac disease in native valve IE (NVE), a lower aged mean patient population in LMICs limits its contribution. ${ }^{1,8,9,18}$

\section{Place of infection acquisition}

The burden of HAIE is growing in HICs, reported in $30 \%$ of IE in recent studies. ${ }^{1,2,18}$ Advances in medical technology, increased indwelling intravascular device use and increased prevalence of comorbidities such as end-stage renal disease contribute to this burden. ${ }^{33}$

Two LMIC studies reported on the site of acquisition of IE. Simsek-Yavuz et al. ${ }^{21}$ characterized $23 \%$ of IE cases as HAIE at a referral centre in Turkey, while Damasco et al. ${ }^{34}$ reported predominantly HAIE (56\%) in two centres in Brazil. In the latter study, an indwelling intravenous catheter was the main source of infection in the entire patient cohort, while among HAIE cases, 55\% had chronic renal insufficiency as a comorbidity. The authors attributed the higher HAIE rates to advances in medical practices in Rio.

\section{Microbial etiology}

Staphylococcus is now the leading causative microorganism of IE in HICs, followed by Streptococcus and Enterococcus. Together, these microorganisms account for $>80 \%$ of the microbial etiology of IE in HICs. ${ }^{1,2,18}$

Describing the microbial etiology of IE in LMICs is extremely challenging as limited data exists on IE as it is, and one's ability to make conclusions from the available data is further curtailed by a high rate of non-identified microorganisms. From available data however, Staphylococcus and Streptococcus are the leading causative microorganisms.

\section{Staphylococcus}


Staphylococcus is increasingly common, and in some instances is the leading cause of IE in LMICs (Table 1). Staphylococcus accounts for $15-50 \%$ of cases with Staphylococcus aureus more common than coagulase negative Staphylococcus (CoNS). ${ }^{19-23,25,31,32,34}$ Comparison with pre-millennial ranges shows an increase in Staphylococcus which was previously reported in less than $20 \%$ of cases. ${ }^{11,13,28}$

The emergence of Staphylococcus as a leading cause of IE in LMICs, a trend that was also previously noted in HICs, may reflect medical technology advances, increased hospital contact and increasing comorbidities. ${ }^{9,34,35}$ Consistent with these factors, it is noteworthy that only UMIC studies reported Staphylococcus as the leading cause of IE, accounting for up to $50 \%$ of cases..$^{21,22,25,31,32,34}$ In lower-middle income countries however, Streptococcus still predominates. ${ }^{19,20}$

\section{Streptococcus}

Streptococcus causes $18-54 \%$ of IE cases, with viridans group streptococci predominating. ${ }^{19-23,25,31,32,34}$ Streptococcus is common among younger patients, patients with community-acquired IE and patients with rheumatic or congenital NVE. ${ }^{19,20,34}$ This finding was highlighted by Simsek-Yavuz et al. ${ }^{21}$ in a study in Turkey where although Staphylococcus was the leading cause of IE in the entire cohort, Streptococcus was significantly more prevalent among patients with a native valve, patients less than 40 years of age, and patients with community-acquired IE.

\section{Other organisms}

Less common causes of IE in LMICs are Enterococcus, gram negative bacteria and true causative agents of blood culture negative IE such as Bartonella and Coxiella. ${ }^{19-22}$ This is consistent with reports from HICs, except for Enterococcus which is the third leading cause of IE in HICs after Staphylococcus and Streptococcus. 
Prevalence of Enterococcal IE was reported to range between $7-18 \%{ }^{19-22,31,32}$ in our reviewed studies. Damasco et al. ${ }^{34}$ however, reported Enterococcus as the second most common cause of IE, after Staphylococcus, accounting for $27 \%$ of cases. Furthermore, among patients aged $\geq 40$ years, Enterococcus was the most commonly isolated microorganism, indicating a predilection for increasing age, a finding supported by SimsekYavuz et al. ${ }^{21}$ who reported increasing prevalence of Enterococcus in individuals $>50$ years old.

In summary, conclusions about the microbial etiology of IE in LMICs are hampered by scarce available data and a high rate of unidentified microorganisms due to widespread prior antibiotic use, limited microbiological capacity and inadequate sampling procedures, challenges that are discussed further later. Inconsistent serological testing for atypical microorganisms further limits etiology determination. ${ }^{36}$ Existing data, however, highlight a prominent contribution of Streptococcus and a growing contribution of Staphylococcus, particularly in UMICs.

\section{TREATMENT}

Prompt and appropriate organism-specific antibiotic therapy is recommended to improve clinical outcomes for IE patients, while surgical intervention is recommended for complicated IE. ${ }^{37-40}$ The antimicrobial regimens employed, mean hospital stay and surgical intervention rates are summarised in Table 1 and discussed below.

\section{Medical therapy}

Medical therapy remains the most common treatment for IE due to limited access to cardiovascular surgery in LMICs. ${ }^{16}$ Setting-specific treatment guidelines are non-existent and subsequently, societal guidelines from HICs are referenced in deciding treatment. ${ }^{37,41}$ Determination of the microbial etiology of IE should be the cornerstone of selecting 
antibiotic therapy; however, as discussed later, this is hampered in LMICs by high rates of organism non-identification.

In the absence of reliable microbiological findings, antibiotic therapy in a majority of suspected cases remains empirical. The choice of therapy varies because the guidelines for empirical therapy from $\mathrm{HICs}^{42}$ may not be translatable to settings with different local susceptibility patterns.

In the few cases where microbial etiology is determined, antibiotics are instituted based on standard guideline based regimens. ${ }^{37,41}$ Our reviewed studies reveal high usage of combination therapy that typically includes beta-lactams or peptide antibiotics and aminoglycosides, with prolonged duration of therapy ranging between 35-67 days. ${ }^{20,43}$

Mean duration of hospital stay ranges between 16-38 days. ${ }^{21,22,34,43}$ Outpatient parenteral antibiotic therapy (OPAT) may be considered in stable patients to decrease hospital stay. ${ }^{44}$ OPAT for IE however, may not be possible in most LMIC settings due to a high rate of complications associated with late hospital presentation, and unavailability of required medical services in the outpatient setting. None of our reviewed studies reported on the rates of OPAT.

\section{Surgical management}

Due to late presentation and/or diagnosis, patients often present with complications, such as valve abscess, systemic embolization, heart failure and hemodynamic instability, which are indications for emergent surgical intervention. Reviewed LMIC studies reveal that surgical intervention rates remains low, ranging from $0-53 \%$ in our reviewed studies. ${ }^{19-}$ 25,31,32,34,43 The higher figures in this range (42-53\%) predominantly come from UMICs. ${ }^{21,22,24,25,32,43}$ These figures refer to surgical rates among the entire patient cohort in the given study, and not among patients with indications for surgical intervention, it is 
therefore difficult to conclude whether intervention rates are optimal. However, in lowermiddle income countries for instance, surgical intervention was less than $15 \%$, despite over $30 \%$ of IE patients presenting in heart failure, an indication for surgery. ${ }^{19,20}$ In a cardiac centre in India however, where access to surgical consult and intervention was prompt, Gupta et al. reported a surgical intervention rate of $49 \%{ }^{23}$

Among patients with complications that require emergent intervention, the optimal timing for surgery remains controversial. ${ }^{45}$ Early surgery however, has been shown to decrease the rate of embolic events ${ }^{46}$ in-hospital, ${ }^{47}$ and 6-month mortality. ${ }^{48}$ Two studies in our review reported on the timing of surgery. Rekik et al. ${ }^{43}$ reported a mean delay to surgery of 15 days among 20 patients with PVE and indications for surgery. Similarly, Trabelsi et al. ${ }^{24}$ reported a mean time between admission and surgery of 16 days among 68 patients with NVE and surgical indications.

\section{CHALLENGES}

The prevailing challenges to the diagnosis and management of IE that co-exist in LMICs are illustrated in Figure 1 and discussed below.

\section{High prevalence of rheumatic heart disease}

The prevalence of RHD remains high and accounts for a significant portion of cardiovascular disease (CVD) morbidity and mortality in LMICs. ${ }^{15,49,50} \mathrm{IE}$ is a common complication of RHD and as highlighted earlier, up to half of IE cases in LMICs are superimposed on RHD. $79 \%$ of the 15.6 million cases of RHD in 2005 were from LMICs, driven by poor standards of living, poor nutritional status, and limited access to healthcare facilities and penicillin prophylaxis. ${ }^{15}$ With recent estimates projecting that up to 80 million

people worldwide have RHD, more LMIC patients are at risk for IE. ${ }^{50,51}$ Addressing the high 
burden of RHD in LMICs is therefore a key step in decreasing IE related morbidity and mortality.

\section{Late hospital presentation}

IE patients in LMICs often present to hospital late. Median time between symptom onset and hospital presentation ranged from 15-36 days in our reviewed studies..$^{22,24,34}$ Consequently, patients frequently presented with IE complications such as heart failure and stroke. ${ }^{19-22}$ IE complications are associated with higher mortality, and require surgical intervention which is often unavailable in LMICs, furthur worsening clinical outcomes.

\section{High rate of organism non-identification}

Identification of causative microorganisms, mainly through blood cultures, is the cornerstone of IE diagnosis and is crucial to the initiation of effective organism-specific antimicrobial therapy. ${ }^{52}$

The rate of organism non-identification in IE from HIC studies is $<5 \%$, while this figure is generally $>35 \%$, reaching upto $60 \%$, in LMICs. ${ }^{1,19,20,24,25,31,43}$ Causes of this high rate are multifactorial. First, widespread use of antibiotics prior to collection of blood samples for culture substantially decreases the likelihood of obtaining growth on culture media. In our reviewed studies, $35-74 \%$ of patients reported prior antibiotic use..$^{20,23,24,32,43}$

Second, there is limited capacity for high quality microbiology studies, particularly in lower-middle income and low-income settings. Blood culture infrastructure is underdeveloped and testing reagents are often unavailable. ${ }^{53}$ Facilities for serological testing of atypical organisms are also unavailable in the poorest settings. ${ }^{36}$ Serological testing increases the rate of organism identification and is particularly useful for organisms responsible for culture-negative IE such as Bartonella henselae, Brucella melitensis and Coxiella burnetii. 
Third, inappropriate procedures in collecting samples for culture may occur. ${ }^{54}$ It is recommended that at least three sets of blood culture samples are collected over a period of 12 hours (or more practically, with the first and last set obtained at least an hour apart) ${ }^{52}$ LMIC studies however, reported that a lower number of samples were collected, resulting in few samples fulfilling major IE criteria for blood culture. ${ }^{20}$

\section{Empiric antibiotic therapy}

Prompt identification of microorganisms and initiation of appropriate antibiotics decreases IE mortality and morbidities such as stroke. ${ }^{37,41}$ In the absence of organism identification in LMICs however, antibiotic therapy in suspected cases is empirical. Choice of therapy varies between regions, hospitals, and physicians. This is because resource poor regions often have little guidance to inform empirical therapy since local data on prevalent microorganisms and antibiotic resistance are scarce..$^{55}$

Additional problems related to antibiotic use include cost limitations in resource poor settings. Physicians and patients have little access to expensive regimens that may be required for drug resistant cases. ${ }^{56}$ Although commonly used antibiotics for IE such as gentamicin, vancomycin and most beta-lactam antibiotics, including penicillin and ceftriaxone, are included in the WHO essential medicines list, agents such as daptomycin which may be necessary in drug resistant IE cases are not. ${ }^{57}$ Antibiotic stock-outs may also occur during the course of treatment, leading to missed drug doses or unnecessary regimen changes.

\section{Little capacity for therapeutic drug monitoring}

Therapeutic drug monitoring (TDM) services are frequently unavailable in LMICs. ${ }^{58}$ TDM is recommended for antibiotics used to manage IE, especially vancomycin and aminoglycosides, to ensure adequate drug levels are reached to maximize efficacy, decrease risk of resistance development, and prevent toxicities such as renal failure. Empiric antibiotic 
therapy often involves combinational agents which further increase risk of nephrotoxicity. ${ }^{59,60}$ Furthermore, among patients with heart failure which commonly complicates IE, the risk of nephrotoxicity is heightened. ${ }^{61}$

\section{Dismal access to surgery}

Early surgery decreases mortality among patients with IE complications. ${ }^{39,47}$ In HICs, approximately half of IE patients in reported prospective series' undergo surgical intervention, ${ }^{1,2,62}$ with up to $75 \%$ of patients with indications for surgery receiving it. ${ }^{62}$ In our reviewed studies, although UMIC studies reported surgical intervention rates close to HICs, two lower middle-income country studies reported low rates of $0 \%$ and $15 \% .{ }^{19,20} \mathrm{We}$ however found no information on the rates of surgical intervention among patients with indications for surgery, however, given the high rate of complications among IE patients in our reviewed studies, we can conclude that this rate was low. Only a few referral facilities in Sub-Saharan Africa (SSA) have the physical and human resource infrastructure to perform cardiovascular surgery, ${ }^{16,63}$ and the few with such capacity often have long waiting lists, making emergent intervention difficult. Furthermore, where such opportunities are available, high costs remain an insurmountable barrier for a majority of patients. ${ }^{14}$

\section{FUTURE DIRECTIONS IN RESEARCH}

A research agenda is urgently required around IE in LMICs. Our review reveals a scarcity of studies documenting clinical experience since the new millennium. In low-income countries for instance, we found no studies meeting our search criteria. A high RHD prevalence in lower income settings, coupled with medical progress in improving economies where new IE risk factors for the population emerge, implies that IE will remain an important CVD in LMICs. In addition, challenges in IE diagnosis and management result in higher IE related morbidity and mortality in LMICs compared to HICs. ${ }^{64}$ Setting-specific data is 
therefore required to track the clinical characteristics, diagnostic and management practices, and outcomes for patients with IE in order to foster investment in improving microbiological, echocardiographic and cardiovascular surgery capacity.

We propose the following steps to stimulate research and further understanding of IE in LMICs. First, where there is a critical lack of published data such as in the low-income countries, retrospective reviews should be conducted based on existing records. This would serve as a first step to provide critical expeditious updates on the state of IE, while building capacity in research, and providing preliminary data that can be used to justify larger prospective studies.

Secondly, prospective IE registries in LMICs need to be established. Current studies are limited by few patient numbers in single healthcare centres which hampers generalizability. Experiences from contemporary registries such as the International Collaboration on Endocarditis (ICE) have effectively improved understanding of IE. ${ }^{1,65}$ This registry however, has predominantly included sites from HICs and UMICs, and to date has not included a site from a low income country. ${ }^{1}$ Experiences from ICE should be leveraged in order to build capacity in LMICs to enable them to form rigorous registries documenting experiences in their settings.

\section{LIMITATIONS OF REVIEW}

Our review has several strengths. Our focus on studies reporting findings from 2000present day provides a contemporary update on the causes of IE in LMICs, and the challenges yet to be addressed. Where possible, we have also described the differences in the IE profile between different LMIC economies.

Our review however, has several limitations. We found few LMIC studies on IE, with a majority of them from UMICs and none from low-income countries. Therefore, this review 
may not adequately reflect findings from the lowest resource settings. Available studies have few patients, mostly from single centres, and are heterogeneous in nature, limiting our ability to form generalizable conclusions. Finally, most of the available data comes from referral centres and thus our findings are subject to referral bias.

\section{CONCLUSION}

Staphylococcus is an increasingly important cause of IE in LMICs, particularly in UMICs although the rate of organism non-identification is high. RHD remains the major underlying cardiac pathology, with a growing contribution of PVE. Rates of access to surgery in UMICs for complicated IE are as high as in HICs, but remain dismal in lower-middle income countries. Publication of retrospective findings in low-income settings, and the formation of collaborative registries to improve understanding of contemporary IE in LMICs must be encouraged. 


\section{References}

1. Murdoch DR, Corey GR, Hoen B, et al. Clinical presentation, etiology, and outcome of infective endocarditis in the 21st century: the International Collaboration on EndocarditisProspective Cohort Study. Archives of internal medicine. 2009;169(5):463-473.

2. Selton-Suty C, Celard M, Le Moing V, et al. Preeminence of Staphylococcus aureus in infective endocarditis: a 1-year population-based survey. Clinical infectious diseases : an official publication of the Infectious Diseases Society of America. 2012;54(9):1230-1239.

3. Thuny F, Avierinos JF, Tribouilloy C, et al. Impact of cerebrovascular complications on mortality and neurologic outcome during infective endocarditis: a prospective multicentre study. European heart journal. 2007;28(9):1155-1161.

4. Ferraris L, Milazzo L, Ricaboni D, et al. Profile of infective endocarditis observed from 2003 2010 in a single center in Italy. BMC infectious diseases. 2013;13:545.

5. Nadji G, Rusinaru D, Remadi JP, Jeu A, Sorel C, Tribouilloy C. Heart failure in left-sided native valve infective endocarditis: characteristics, prognosis, and results of surgical treatment. European journal of heart failure. 2009;11(7):668-675.

6. San Roman JA, Lopez J, Vilacosta I, et al. Prognostic stratification of patients with left-sided endocarditis determined at admission. The American journal of medicine. 2007;120(4):369.e361-367.

7. Murray CJ, Vos T, Lozano R, et al. Disability-adjusted life years (DALYs) for 291 diseases and injuries in 21 regions, 1990-2010: a systematic analysis for the Global Burden of Disease Study 2010. Lancet (London, England). 2012;380(9859):2197-2223.

8. Coffey S, Cairns BJ, lung B. The modern epidemiology of heart valve disease. Heart. 2016;102(1):75-85.

9. Yew HS, Murdoch DR. Global trends in infective endocarditis epidemiology. Current infectious disease reports. 2012;14(4):367-372.

10. Bennis A, Zahraoui M, Azzouzi L, et al. [Bacterial endocarditis in Morocco]. Annales de cardiologie et d'angeiologie. 1995;44(7):339-344.

11. Garg N, Kandpal B, Garg N, et al. Characteristics of infective endocarditis in a developing country-clinical profile and outcome in 192 Indian patients, 1992-2001. International journal of cardiology. 2005;98(2):253-260.

12. Ifere OA, Masokano KA. Infective endocarditis in children in the Guinea savannah of Nigeria. Annals of tropical paediatrics. 1991;11(3):233-240.

13. Koegelenberg CF, Doubell AF, Orth H, Reuter H. Infective endocarditis in the Western Cape Province of South Africa: a three-year prospective study. QJM : monthly journal of the Association of Physicians. 2003;96(3):217-225.

14. Tariq M, Alam M, Munir G, Khan MA, Smego RA, Jr. Infective endocarditis: a five-year experience at a tertiary care hospital in Pakistan. International journal of infectious diseases : IIID : official publication of the International Society for Infectious Diseases. 2004;8(3):163170.

15. Carapetis JR, Steer AC, Mulholland EK, Weber M. The global burden of group A streptococcal diseases. The Lancet. Infectious diseases. 2005;5(11):685-694.

16. Mocumbi AO. The challenges of cardiac surgery for African children. Cardiovascular Journal of Africa. 2012;23(3):165-167.

17. World Bank Country and Lending Groups. https://datahelpdesk.worldbank.org/knowledgebase/articles/906519. Accessed 06/01, 2016.

18. Sy RW, Kritharides L. Health care exposure and age in infective endocarditis: results of a contemporary population-based profile of 1536 patients in Australia. European heart journal. 2010;31(15):1890-1897. 
19. Mirabel M, Rattanavong S, Frichitthavong K, et al. Infective endocarditis in the Lao PDR: clinical characteristics and outcomes in a developing country. International journal of cardiology. 2015;180:270-273.

20. Math RS, Sharma G, Kothari SS, et al. Prospective study of infective endocarditis from a developing country. American heart journal. 2011;162(4):633-638.

21. Simsek-Yavuz S, Sensoy A, Kasikcioglu H, et al. Infective endocarditis in Turkey: aetiology, clinical features, and analysis of risk factors for mortality in 325 cases. International journal of infectious diseases : IJID : official publication of the International Society for Infectious Diseases. 2015;30:106-114.

22. Nunes MCP, Gelape CL, Ferrari TCA. Profile of infective endocarditis at a tertiary care center in Brazil during a seven-year period: prognostic factors and in-hospital outcome. International Journal of Infectious Diseases. 2010;14(5):e394-e398.

23. Gupta A, Gupta A, Kaul U, Varma A. Infective endocarditis in an Indian setup: Are we entering the 'modern' era? Indian Journal of Critical Care Medicine : Peer-reviewed, Official Publication of Indian Society of Critical Care Medicine. 2013;17(3):140-147.

24. Trabelsi I, Rekik S, Znazen A, et al. Native valve infective endocarditis in a tertiary care center in a developing country (Tunisia). The American journal of cardiology. 2008;102(9):12471251.

25. Nel SH, Naidoo DP. An echocardiographic study of infective endocarditis, with special reference to patients with HIV. Cardiovascular Journal of Africa. 2014;25(2):50-57.

26. Zuhlke LJ, Engel ME, Watkins D, Mayosi BM. Incidence, prevalence and outcome of rheumatic heart disease in South Africa: a systematic review of contemporary studies. International journal of cardiology. 2015;199:375-383.

27. Cetinkaya $\mathrm{Y}$, Akova $\mathrm{M}$, Akalin $\mathrm{HE}$, et al. A retrospective review of 228 episodes of infective endocarditis where rheumatic valvular disease is still common. International journal of antimicrobial agents. 2001;18(1):1-7.

28. Letaief A, Boughzala E, Kaabia N, et al. Epidemiology of infective endocarditis in Tunisia: a 10-year multicenter retrospective study. International journal of infectious diseases : IJID : official publication of the International Society for Infectious Diseases. 2007;11(5):430-433.

29. Remenyi B, Carapetis J, Wyber R, Taubert K, Mayosi BM. Position statement of the World Heart Federation on the prevention and control of rheumatic heart disease. Nature reviews. Cardiology. 2013;10(5):284-292.

30. Seckeler MD, Hoke TR. The worldwide epidemiology of acute rheumatic fever and rheumatic heart disease. Clinical epidemiology. 2011;3:67-84.

31. Elbey MA, Akdag S, Kalkan ME, et al. A multicenter study on experience of 13 tertiary hospitals in Turkey in patients with infective endocarditis. Anadolu kardiyoloji dergisi : $A K D=$ the Anatolian journal of cardiology. 2013;13(6):523-527.

32. Leblebicioglu $\mathrm{H}$, Yilmaz $\mathrm{H}$, Tasova $\mathrm{Y}$, et al. Characteristics and analysis of risk factors for mortality in infective endocarditis. European journal of epidemiology. 2006;21(1):25-31.

33. Cahill TJ, Prendergast BD. Infective endocarditis. Lancet (London, England). 2016;387(10021):882-893.

34. Damasco PV, Ramos JN, Correal JC, et al. Infective endocarditis in Rio de Janeiro, Brazil: a 5year experience at two teaching hospitals. Infection. 2014;42(5):835-842.

35. Fowler VG, Jr., Miro JM, Hoen B, et al. Staphylococcus aureus endocarditis: a consequence of medical progress. Jama. 2005;293(24):3012-3021.

36. Rattanavong S, Fournier PE, Chu V, et al. Bartonella henselae endocarditis in Laos - 'the unsought will go undetected'. PLoS neglected tropical diseases. 2014;8(12):e3385.

37. Baddour LM, Wilson WR, Bayer AS, et al. Infective Endocarditis in Adults: Diagnosis, Antimicrobial Therapy, and Management of Complications: A Scientific Statement for Healthcare Professionals From the American Heart Association. Circulation.

2015;132(15):1435-1486. 
38. Habib G, Lancellotti P, Antunes MJ, et al. 2015 ESC Guidelines for the management of infective endocarditis: The Task Force for the Management of Infective Endocarditis of the European Society of Cardiology (ESC)Endorsed by: European Association for Cardio-Thoracic Surgery (EACTS), the European Association of Nuclear Medicine (EANM). European heart journal. 2015;36(44):3075-3128.

39. Kiefer T, Park L, Tribouilloy C, et al. Association between valvular surgery and mortality among patients with infective endocarditis complicated by heart failure. Jama. 2011;306(20):2239-2247.

40. Kang DH, Kim YJ, Kim SH, et al. Early surgery versus conventional treatment for infective endocarditis. The New England journal of medicine. 2012;366(26):2466-2473.

41. Habib G, Lancellotti P, Antunes MJ, et al. 2015 ESC Guidelines for the management of infective endocarditis. <div xmlns="http://www.w3.org/1999/xhtml"><span class="subtitle" $>$ The Task Force for the Management of Infective Endocarditis of the European Society of Cardiology (ESC)<br/>Endorsed by: European Association for CardioThoracic Surgery (EACTS), the European Association of Nuclear Medicine (EANM)</span ></div>. 2015.

42. Gould FK, Denning DW, Elliott TS, et al. Guidelines for the diagnosis and antibiotic treatment of endocarditis in adults: a report of the Working Party of the British Society for Antimicrobial Chemotherapy. The Journal of antimicrobial chemotherapy. 2012;67(2):269289.

43. Rekik S, Trabelsi I, Znazen A, et al. Prosthetic valve endocarditis: management strategies and prognosis: A ten-year analysis in a tertiary care centre in Tunisia. Netherlands heart journal : monthly journal of the Netherlands Society of Cardiology and the Netherlands Heart Foundation. 2009;17(2):56-60.

44. Tice AD, Rehm SJ, Dalovisio JR, et al. Practice Guidelines for Outpatient Parenteral Antimicrobial Therapy. Clinical Infectious Diseases. 2004;38(12):1651-1671.

45. Bannay A, Hoen B, Duval X, et al. The impact of valve surgery on short- and long-term mortality in left-sided infective endocarditis: do differences in methodological approaches explain previous conflicting results? European heart journal. 2011;32(16):2003-2015.

46. Kang D-H, Kim Y-J, Kim S-H, et al. Early Surgery versus Conventional Treatment for Infective Endocarditis. New England Journal of Medicine. 2012;366(26):2466-2473.

47. Lalani T, Cabell CH, Benjamin DK, et al. Analysis of the impact of early surgery on in-hospital mortality of native valve endocarditis: use of propensity score and instrumental variable methods to adjust for treatment-selection bias. Circulation. 2010;121(8):1005-1013.

48. Vikram HR, Buenconsejo J, Hasbun R, Quagliarello VJ. Impact of valve surgery on 6-month mortality in adults with complicated, left-sided native valve endocarditis: a propensity analysis. Jama. 2003;290(24):3207-3214.

49. Celermajer DS, Chow CK, Marijon E, Anstey NM, Woo KS. Cardiovascular disease in the developing world: prevalences, patterns, and the potential of early disease detection. Journal of the American College of Cardiology. 2012;60(14):1207-1216.

50. Nulu K. Rheumatic Heart Disease: The Unfinished Global Agenda. Cardiology clinics. 2016. 51. Paar JA, Berrios NM, Rose JD, et al. Prevalence of rheumatic heart disease in children and young adults in Nicaragua. The American journal of cardiology. 2010;105(12):1809-1814.

52. Li JS, Sexton DJ, Mick N, et al. Proposed modifications to the Duke criteria for the diagnosis of infective endocarditis. Clinical infectious diseases : an official publication of the Infectious Diseases Society of America. 2000;30(4):633-638.

53. Archibald LK, Reller LB. Clinical microbiology in developing countries. Emerging infectious diseases. 2001;7(2):302-305.

54. Mermel LA, Maki DG. Detection of bacteremia in adults: consequences of culturing an inadequate volume of blood. Annals of internal medicine. 1993;119(4):270-272. 
55. Alsan M, Schoemaker L, Eggleston K, Kammili N, Kolli P, Bhattacharya J. Out-of-pocket health expenditures and antimicrobial resistance in low-income and middle-income countries: an economic analysis. The Lancet. Infectious diseases. 2015;15(10):1203-1210.

56. Securing Ugandans' Right To Essential Medicines (SURE). 2011.

57. 19th WHO Model List of Essential Medicines (April 2015).

http://www.who.int/medicines/publications/essentialmedicines/EML2015 8-May-15.pdf. Accessed 08/09, 2016.

58. Nwobodo N. Therapeutic drug monitoring in a developing nation: a clinical guide. JRSM Open. 2014;5(8).

59. Cosgrove SE, Vigliani GA, Fowler VG, Jr., et al. Initial low-dose gentamicin for Staphylococcus aureus bacteremia and endocarditis is nephrotoxic. Clinical infectious diseases : an official publication of the Infectious Diseases Society of America. 2009;48(6):713-721.

60. Burgess LD, Drew RH. Comparison of the incidence of vancomycin-induced nephrotoxicity in hospitalized patients with and without concomitant piperacillin-tazobactam. Pharmacotherapy. 2014;34(7):670-676.

61. Sarraf M, Masoumi A, Schrier RW. Cardiorenal Syndrome in Acute Decompensated Heart Failure. Clinical Journal of the American Society of Nephrology. 2009;4(12):2013-2026.

62. Chu VH, Park LP, Athan E, et al. Association between surgical indications, operative risk, and clinical outcome in infective endocarditis: a prospective study from the International Collaboration on Endocarditis. Circulation. 2015;131(2):131-140.

63. Zühlke L, Mirabel M, Marijon E. Congenital heart disease and rheumatic heart disease in Africa: recent advances and current priorities. Heart. 2013;99(21):1554-1561.

64. Thuny F, Grisoli D, Collart F, Habib G, Raoult D. Management of infective endocarditis: challenges and perspectives. Lancet (London, England). 2012;379(9819):965-975.

65. Cabell CH, Abrutyn E. Progress toward a global understanding of infective endocarditis. Lessons from the International Collaboration on Endocarditis. Cardiology clinics. 2003;21(2):147-158. 